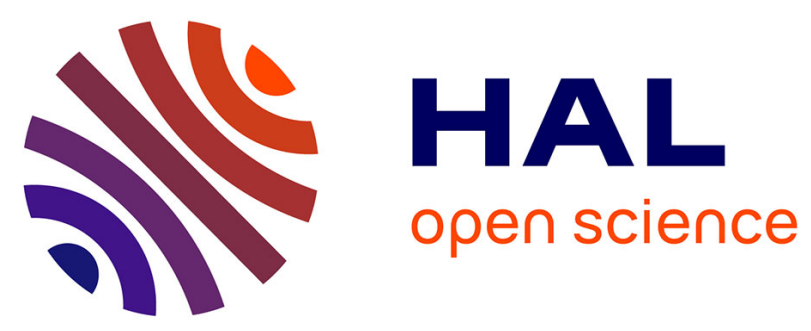

\title{
Pulleys and Force Sensors Influence on Payload Estimation of Cable-Driven Parallel Robots
}

\author{
Etienne Picard, Stéphane Caro, Fabien Claveau, Franck Plestan
}

\section{To cite this version:}

Etienne Picard, Stéphane Caro, Fabien Claveau, Franck Plestan. Pulleys and Force Sensors Influence on Payload Estimation of Cable-Driven Parallel Robots. 2018 IEEE/RSJ International Conference on Intelligent Robots and Systems (IROS 2018), Oct 2018, Madrid, Spain. 10.1109/IROS.2018.8594171 . hal-01862015

\section{HAL Id: hal-01862015 https://hal.science/hal-01862015}

Submitted on 26 Aug 2018

HAL is a multi-disciplinary open access archive for the deposit and dissemination of scientific research documents, whether they are published or not. The documents may come from teaching and research institutions in France or abroad, or from public or private research centers.
L'archive ouverte pluridisciplinaire HAL, est destinée au dépôt et à la diffusion de documents scientifiques de niveau recherche, publiés ou non, émanant des établissements d'enseignement et de recherche français ou étrangers, des laboratoires publics ou privés. 


\title{
Pulleys and Force Sensors Influence on Payload Estimation of Cable-Driven Parallel Robots
}

\author{
Étienne Picard $^{1,2}$, Stéphane Caro ${ }^{2,3}$, Fabien Claveau ${ }^{2,4}$ and Franck Plestan ${ }^{2,5}$
}

\begin{abstract}
The subject of this paper is about the use of a suspended Cable-Driven Parallel Robot (CDPR) for pickand-place operations of heavy and heterogeneous objects. The knowledge of the payload mass and its center of mass in realtime is an asset for robust control of the device, which is required to ensure a good stability, especially when the objects have different shapes, sizes and masses. Accordingly, this paper aims at experimentally evaluating the effects of $(i)$ the pulleys modeling and (ii) the use of force sensors for the payload estimation. It turns out that the consideration of the pulleys into the geometric model of the robot improves the mass and center of mass estimations of the payload. A comparison is made between the estimation of cable tensions from force sensors and from motor currents. Finally, a torque controller with a feedforward term for real-time mass compensation is proposed and implemented on a CDPR prototype.
\end{abstract}

\section{INTRODUCTION}

Cable-Driven Parallel Robots (CDPRs) form a particular class of parallel robots whose moving platform is connected to a fixed base frame by cables. The cables are coiled on motorized winches. Passive pulleys may guide the cables from the winches to the cable exit points. Accordingly, the motion of the platform is controlled by modifying the cable lengths. CDPRs have several advantages such as a relatively low mass of moving parts and a potential large workspace. As a consequence, they can be used in several applications such as heavy payload handling, painting and large structures sandblasting [1], cargo handling [2], warehouse applications [3], large-scale assembly and handling operations [4], and fast pick-and-place operations [5]. Other possible applications include the broadcasting of sporting events, haptic devices [6], support structures for giant telescopes [7], and search and rescue deployable platforms [8]. Some recent works have dealt with the design and reconfiguration planning of reconfigurable CDPRs that can be used in cluttered industrial environments [1].

In pick and place applications, the knowledge of the payload mass and center of gravity is useful to control the

\footnotetext{
*This work is supported by IRT Jules Verne (French Institute in Research and Technology in Advanced Manufacturing Technologies for Composite, Metallic and Hybrid Structures) in the framework of ROCKET project.

1 IRT Jules Verne, Chemin du Chaffault, 44340 Bouguenais, France etienne.picardeirt-jules-verne.fr

${ }^{2}$ Laboratoire des Sciences du Numérique de Nantes, UMR CNRS 6004, 1, rue de la Noë, 44321 Nantes, France

${ }^{3}$ Centre National de la Recherche Scientifique (CNRS), 1, rue de la Noë, 44321 Nantes, France stephane.caro@ls2n.fr

4 IMT-Atlantique (site de Nantes), La Chantrerie, 4 Rue Alfred Kastler, BP 20722, 44307 Nantes Cedex, France fabien. claveaulimt-atlantique.fr

5 Ecole Centrale de Nantes, 1, rue de la Noë, 44321 Nantes, France franck.plestan@ec-nantes.fr
}

platform position accurately and ensure its stability, especially for heavy loads. A relationship between the tension of the cables and the payload characteristics was proposed in [9] for a position based control method able to compensate for cable elasticity, and tested on up to $40 \mathrm{~kg}$ payloads. Elastogeometrical model of CDPRs [10] can be used to calculate the cable length changes under load but their computation cost can be high for real-time implementation in a controller. However, in order to get a good estimation of the payload we had better use a detailed robot model of the robot and the most reliable measurements for cable tensions. Previous works such as [11] and [12] proposed rigid CDPR models including pulleys with two degrees of freedom (DoF) and analyzed the effect of the pulleys onto the workspace size.

This paper deals with the analysis of the effect of the pulley geometric parameters onto the payload estimation as well as the comparison between dynamometers (force sensors) and motor currents to assess the cable tensions. Moreover, a control scheme including a feedforward term for payload mass estimation and compensation in real-time is proposed and implemented on the IRT Jules Verne CAROCA CDPR prototype.

The paper is organized as follows. Section II describes the moving-platform, the available measurements and the test trajectory used in the experiments. Section III expresses the CDPR models with and without the pulleys geometry as well as the payload mass and center of mass estimation method. Section IV presents a torque control scheme suitable for a unknown payload. Section V deals with the influence of the pulleys on the mass and center of mass estimations and a comparison between the results obtained from tension measured by the dynamometers versus tension estimations from the motor torques. To this end, experimental measures along the test trajectory are analyzed. Finally, conclusions are drawn and future work is presented in Sec. VI.

\section{PRototyPE AND EXPERIMENTAL SETUP}

This section presents the CDPR prototype for the experimental validations. This prototype is named CAROCA [1] and shown in Fig. 1.

\section{A. Hardware}

CAROCA is a reconfigurable CDPR developed at IRT Jules Verne for industrial applications experimentation.

This prototype has pulleys of radius $150 \mathrm{~mm}$ with twoDoF that can be displaced in a discrete manner on its frame. Thanks to its reconfigurability, the robot can be assembled 


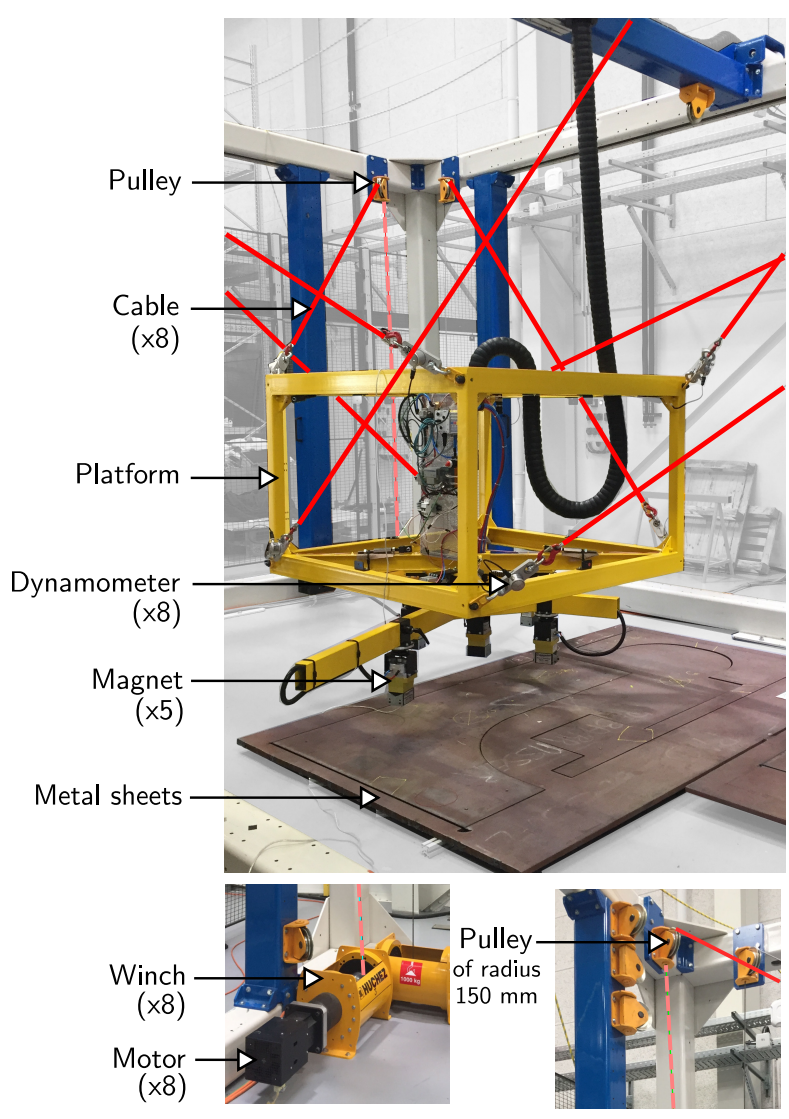

Fig. 1: Top. The moving-platform (MP) equipped with five magnets to pick metal parts and eight dynamometers to measure cable tensions. Bottom. A motor, a winch and a pulley.

both in a suspended or fully-constrained configuration depending on the application at hand. The size of the prototype is $7 \mathrm{~m}$ long $\times 4 \mathrm{~m}$ wide $\times 3 \mathrm{~m}$ high. It is composed of eight cables coiled around $120 \mathrm{~mm}$ diameter Huchez ${ }^{\mathrm{TM}}$ winches, which are pulling a moving-platform (MP). The winches are actuated by synchronous motors of nominal speed and nominal torques equal to $2200 \mathrm{rpm}$ and $15.34 \mathrm{Nm}$, respectively. A two-stage gearbox of reduction ratio equal to 40 is mounted between each motor and each winch. As a consequence, the prototype is capable of lifting up to one ton.

This work is part of the ROCKET project, which aims at displacing metal parts of highly variable shapes and mass, up to $700 \mathrm{~kg}$, thanks to a robust control scheme. The MP size is $1.5 \mathrm{~m} \times 1.5 \mathrm{~m} \times 1 \mathrm{~m}$ and its mass equals to $366 \mathrm{~kg}$ (Fig. 1). Five magnets are embedded under the moving platform to pick metal parts.

\section{B. Measurements}

The robot is equipped with Tractel $^{\mathrm{TM}}$ dynamometers located between the cables and the anchor points of the platform to give a direct measurement of the efforts applied by the cables onto the platform, as shown in Fig. 1. These sensors output a current of magnitude between 4 and $20 \mathrm{~mA}$ proportional to the tension in the corresponding cable, up to $25000 \mathrm{~N}$. They have been calibrated for the typical working range of the robot comprised between 0 and $5000 \mathrm{~N}$ while using a reference force sensor.

Hardware such as motors and control board are standard industrial components commercialized by $\mathrm{B} \& \mathrm{R}$ Automation $^{\mathrm{TM}}$. Angular positions and velocities are available for each motor and the delivered torques are assessed from the motor currents. The robot programming is done in Automation Studio $4.1^{\mathrm{TM}}$, with the code running in a $10 \mathrm{~ms}$ real-time cyclic. The control schemes of the robot are developed and simulations are performed with MATLAB ${ }^{\circledR}$ and Simulink ${ }^{\circledR}$.

In the absence of external sensors and because of the complexity of the direct geometric model in CDPR, no information on the actual pose of the platform is available. However, in order to calculate position errors, it is possible to estimate the position and orientation of the MP by solving the direct geometric model through a Least Squares Method, as presented in [13], for example using the Isqnonlin function in MATLAB.

\section{Test trajectory}

The trajectory presented in Fig. 2 and Fig. 3 simulates a typical pick-and-place operation corresponding to the targeted application, and was used as a basis in the results presented in Sec. V. The platform takes 30 seconds to move from point $\mathrm{A}$ to point $\mathrm{E}$, then 30 seconds from $\mathrm{E}$ back to $\mathrm{A}$.
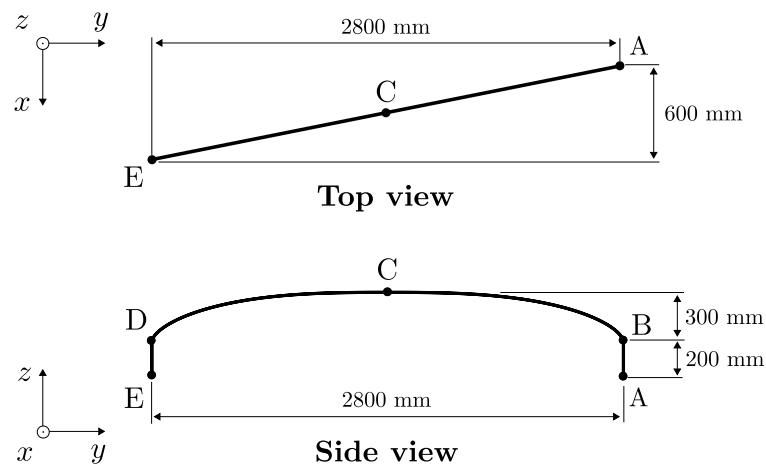

Fig. 2: Top and side views of the test trajectory.

The trajectory is generated using s-curves, which ensure continuous velocity and acceleration trajectory profiles [14]. This test is first performed on the CDPR with the empty MP. In order to evaluate the robustness of the task toward variable payloads, the platform is then loaded by an additional mass of either $122 \mathrm{~kg}$ or $249 \mathrm{~kg}$, denoted as $\mathrm{M}_{1}$ and $\mathrm{M}_{2}$, respectively. Note that the mass variation is significant, namely $+33 \%$ and $+68 \%$ with respect to the MP mass.

\section{MODELING}

The geometric, kinematic and dynamic models of CAROCA are written thereafter. 


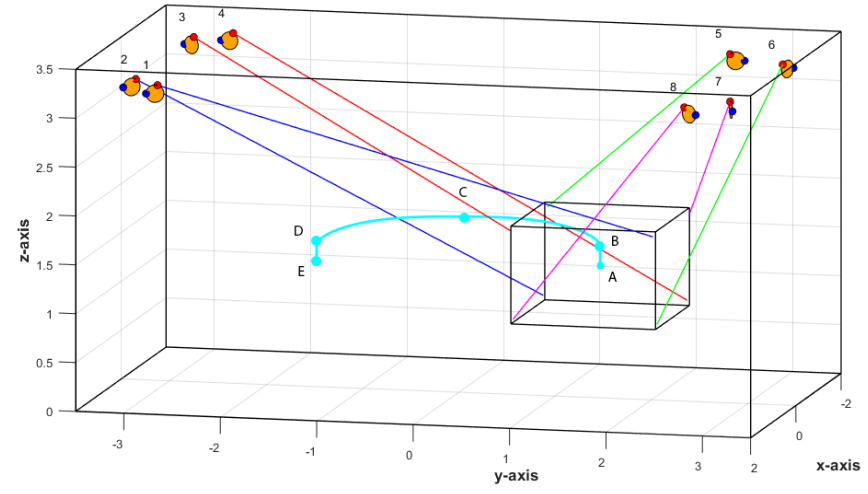

Fig. 3: Test trajectory in blue and CDPR prototype configuration with pulleys in orange.

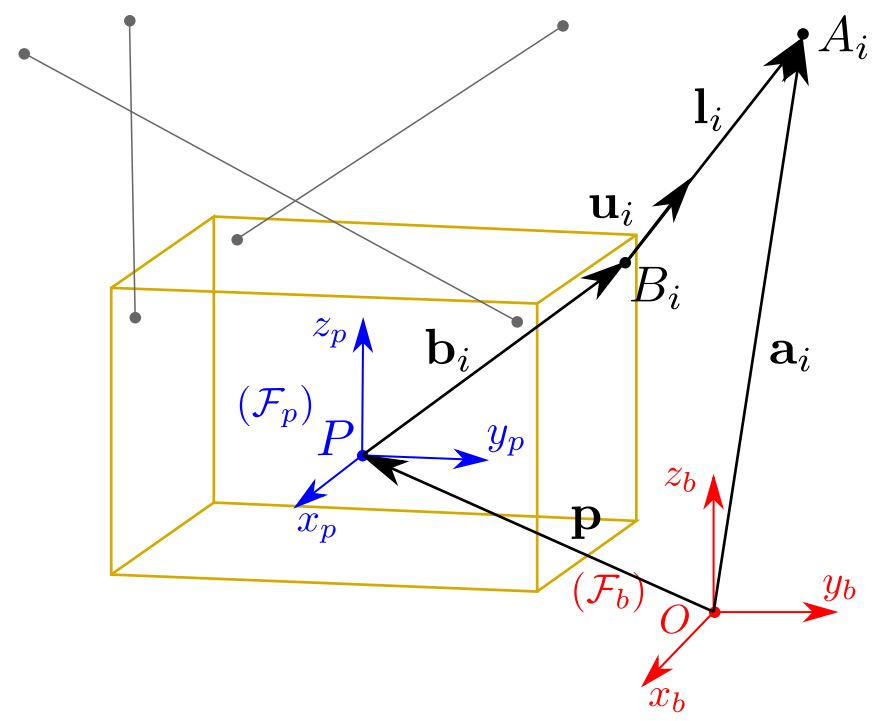

Fig. 4: CDPR geometric parameterization.

\section{A. Model without pulleys}

Fig. 4 depicts the main geometric parameters of a CDPR and its $i^{\text {th }}$ loop-closure equation, $i \in\{1, \ldots, m\}, m$ being the number of cables attached to the MP. $\mathscr{F}_{b}$ is the robot base frame, and $\mathscr{F}_{p}$ is the MP frame.

Cable exit points are denoted as $A_{i}$, while cable anchor points are denoted as $B_{i}$. Vector ${ }^{b} \mathbf{a}_{i}$ points from point $O$ to point $A_{i}$ and is expressed in frame $\mathscr{F}_{b}$. Vector ${ }^{p} \mathbf{b}_{i}$ point from point $P$ to point $B_{i}$ and is expressed in frame $\mathscr{F}_{p}$. Vector ${ }^{b} \mathbf{p}$ is the position vector of point $P$, the MP geometric center, expressed in $\mathscr{F}_{b}$.

In a simplified model, the pulleys are treated as points and the points $A_{i}$ are considered fixed in the frame $\mathscr{F}_{b}$. Their exact position is usually set to the intersection between the vertical rotation axis of the pulley and the horizontal plane containing the axis of the pulley sheave, as depicted in Fig. 5

Vector $\mathbf{l}_{i}$ represents the $i^{t h}$ cable vector and points from $B_{i}$ to $A_{i}$ :

$$
{ }^{b} \mathbf{I}_{i}=l_{i}{ }^{b} \mathbf{u}_{i}={ }^{b} \mathbf{a}_{i}-{ }^{b} \mathbf{p}-{ }^{b} \mathbf{R}_{p}{ }^{p} \mathbf{b}_{\mathbf{i}}={ }^{b} \mathbf{a}_{i}-{ }^{b} \mathbf{p}-{ }^{b} \mathbf{b}_{\mathbf{i}}
$$

with ${ }^{b} \mathbf{R}_{p}$ the rotation matrix from frame $\mathscr{F}_{b}$ to frame $\mathscr{F}_{p}$. $l_{i}$ is the length of the $i^{\text {th }}$ cable and $\mathbf{u}_{i}$ is the unit vector of the $i^{\text {th }}$ cable vector, defined as

$$
l_{i}=\left\|\mathbf{l}_{i}\right\|_{2} \quad \quad \mathbf{u}_{i}=\frac{\mathbf{l}_{i}}{\left\|\mathbf{l}_{i}\right\|_{2}}
$$

where $\|.\|_{2}$ denotes the Euclidean norm of a vector.

The static equilibrium of the platform is given by

$$
\mathbf{W}_{I} \mathbf{t}+\mathbf{w}_{e}+\mathbf{w}_{g}=0
$$

$\mathbf{W}_{I}$ being named as the wrench matrix of the robot [15] and expressed as

$$
\mathbf{W}_{I}=\left[\begin{array}{ccccc}
\mathbf{u}_{1} & \ldots & \mathbf{u}_{i} & \ldots & \mathbf{u}_{m} \\
{ }^{b} \mathbf{b}_{1} \times \mathbf{u}_{1} & \ldots & { }^{b} \mathbf{b}_{i} \times \mathbf{u}_{i} & \ldots & { }^{b} \mathbf{b}_{m} \times \mathbf{u}_{m}
\end{array}\right]
$$

$\mathbf{t}$ is the cable tension vector, $\mathbf{w}_{g}$ is the wrench applied to the platform due to gravity and $\mathbf{w}_{e}$ is an external wrench expressed in the fixed reference frame $\mathscr{F}_{b}$.

The kinematic Jacobian matrix $\mathbf{J}$ of the CDPR is obtained from $\mathbf{W}_{I}$ as:

$$
\mathbf{J}=-\mathbf{W}_{I}^{T}
$$

\section{B. Model including pulleys}

To improve the accuracy of the pulley geometry, we introduce $A_{i}^{\prime}$ as the moving exit point of the cable onto the pulley sheave. The parameterization for a two-DoF pulley is given in Fig. 5. The $i^{\text {th }}$ cable is tangent to its pulley at point $A_{i}^{\prime} . l_{c i}$ is the distance from point $A_{i}^{\prime}$ to point $B_{i} . l_{p i}$ is the length of the $i^{t h}$ cable part embracing the shape of the $i^{\text {th }}$ pulley sheave. Point $A_{i}$ is the intersection point between the vertical rotation axis of the $i^{\text {th }}$ pulley and the horizontal plane containing the axis of its sheave.

Details about the calculations of the position of $A_{i}^{\prime}$ and angles $\theta_{i}, \alpha_{i}, \beta_{i}$ and $\gamma_{i}$ can be found in [11] and [12]. We now write:

$$
{ }^{b} \mathbf{o}_{i}={ }^{b} \mathbf{a}_{i}+r_{p}{ }^{b} \mathbf{x}_{p} \quad{ }^{b} \mathbf{x}_{p}={ }^{b} \mathbf{R}_{\text {pulley }}{ }^{p} \mathbf{x}_{p}
$$

where ${ }^{b} \mathbf{R}_{\text {pulley }}$ is the rotation matrix between $\mathscr{F}_{b}$ and a new frame $\mathscr{F}_{\text {pulley }}$ attached to the pulley and $r_{p}$ is the inner radius of the sheave groove.

By including the pulleys geometry, we obtain a new wrench matrix $\mathbf{W}_{I I}$ :

$$
\mathbf{W}_{I I}=\left[\begin{array}{ccccc}
\mathbf{e}_{1} & \ldots & \mathbf{e}_{i} & \ldots & \mathbf{e}_{m} \\
{ }^{b} \mathbf{b}_{1} \times \mathbf{e}_{1} & \ldots & { }^{b} \mathbf{b}_{i} \times \mathbf{e}_{i} & \ldots & { }^{b} \mathbf{b}_{m} \times \mathbf{e}_{m}
\end{array}\right]
$$

where $\mathbf{e}_{i}$ is the new unit vector of the $i^{\text {th }}$ cable vector, defined as:

$$
l_{c i}=\left\|\mathbf{l}_{c i}\right\|_{2} \quad \mathbf{e}_{i}=\frac{\mathbf{l}_{c i}}{\left\|\mathbf{l}_{c i}\right\|_{2}}
$$

with the cable vector ${ }^{b} \mathbf{l}_{c i}$ from $A_{i}^{\prime}$ to $B_{i}$

$$
{ }^{b} \mathbf{l}_{c i}={ }^{b} \mathbf{a}_{i}-r_{p}\left({ }^{b} \mathbf{z}_{p}+{ }^{b} \mathbf{e}_{i}\right) \times{ }^{b} \mathbf{y}_{p}-{ }^{b} \mathbf{b}_{i}
$$




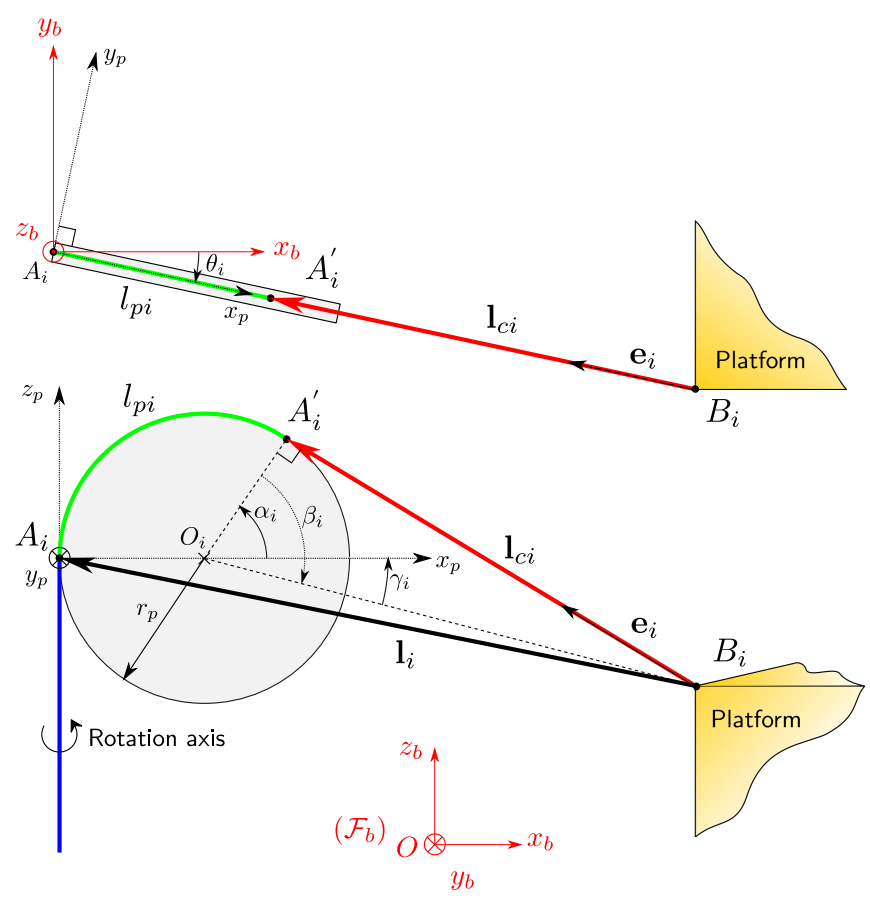

Fig. 5: Top and side views of a two-DoF pulley.

In what remains, Model I stands for the geometric CDPR model obtained by neglecting the pulleys (see Sec. III.A) whereas Model II stands for the geometric CDPR model obtained by considering the pulleys.

\section{Dynamic model}

From [15], the dynamic model of a CDPR can be written as

$$
\mathbf{W t}-\mathbb{I}_{p} \dot{\mathbf{v}}-\mathbf{C v}+\mathbf{w}_{e}+\mathbf{w}_{g}=0
$$

with $\mathbb{I}_{p}$ the spatial inertia of the platform and $\mathbf{C}$ the matrix of the centrifugal and Coriolis wrenches. In what remains, the wrench matrix is either $\mathbf{W}_{I}$ or $\mathbf{W}_{I I}$ depending on the geometric model used in the control scheme.

Given that the center of mass of the platform, denoted as $G$, does not coincide with the origin of frame $\mathscr{F}_{p}$, the wrench $\mathbf{w}_{g}$ due to the gravity acceleration $\mathbf{g}=[0,0,-g]$ is defined as

$$
\mathbf{w}_{g}=\left[\begin{array}{c}
m \mathbf{I}_{3} \\
\mathbf{M} \hat{\mathbf{S}}_{p}
\end{array}\right] \mathbf{g}
$$

with $m$ the mass of the platform, $g=9.81 \mathrm{~m} \cdot \mathrm{s}^{-2}, \mathbf{I}_{3}$ the $3 \times 3$ identity matrix, $\mathbf{M S}_{p}={ }^{b} \mathbf{R}_{p}\left[M x_{p}, M y_{p}, M z_{p}\right]^{\mathrm{T}}$ the first momentum of the moving platform defined with respect to frame $\mathscr{F}_{b}$. The vector $\mathbf{S}_{p}=\left[x_{p}, y_{p}, z_{p}\right]^{\mathrm{T}}$ defines the position of $G$ in $\mathscr{F}_{p}$. M $\hat{\mathbf{S}}_{p}$ is the skew-symmetric matrix associated to $\mathbf{M S}_{p}$. Note that the other terms of (10) are detailed in [15].

\section{Payload mass $(m)$ and center of mass $\left(x_{g}, y_{g}\right)$ estimation}

We describe here a method to obtain a CDPR payload mass and center of mass, knowing the cable tensions.

The following assumptions are made:

1) the orientation of the moving platform is constant
2) the linear velocity of the moving-platform is low

3) the only wrench applied to the platform is due to the carried payload, namely, the metal plate

4) the cables are straight and inelastic

These hypothesis are acceptable in our pick-and-place application of heavy parts, where it is desirable to keep the platform leveled and the dynamics of the trajectories low. It should be noted that a similar approach is presented in [9], but with cables in polymer and lighter payloads.

Equation (10) can then be rewritten as:

$$
\mathbf{W t}+\mathbf{w}_{g}=0
$$

From (11) and (12), $\mathbf{w}_{g}$ can be expressed as:

$$
\mathbf{w}_{g}=\left[\begin{array}{c}
0 \\
0 \\
-m g \\
-m g y_{G} \\
m g x_{G} \\
0
\end{array}\right]=-\mathbf{W t}=-\mathbf{W}\left[\begin{array}{c}
t_{1} \\
\vdots \\
t_{i} \\
\vdots \\
t_{8}
\end{array}\right]
$$

Knowing the tensions $\mathbf{t}$ in each cable and the wrench matrix $\mathbf{W}$, it is then possible to calculate the payload mass $m$ and the Cartesian coordinates $x_{G}$ and $y_{G}$ expressed in $\mathscr{F}_{p}$ of the center of mass of the set composed of the MP and the carried payload.

Cable tensions can be either measured using force sensors or estimated from the motor currents as discussed in Sec. V. The wrench matrix $\mathbf{W}$ depends from the platform position. As explained in Sec. II-B it is only possible to estimate the MP pose by solving the direct geometric model with Least Squares Method. However, the computation cost and the uncertainties can be detrimental for a real-time mass estimation method. As a consequence, in this paper the errors between real and desired platform pose are supposed to be small enough along the trajectory in order to use the desired positions in the calculation of $\mathbf{W}$.

\section{TORQUE CONTROLLER WITH REAL-TIME MASS COMPENSATION}

From the evaluation of the payload mass, a new controller with a compensation term updated with the real-time estimation is presented thereafter.

The control scheme implemented, depicted in Fig. 6, is based on a proportional-derivative strategy (PD) that is well established in robotics including CDPRs [16], with $\mathbf{X}_{d}$ the 6dimensional vector containing the desired Cartesian position and orientation of MP, $\dot{\mathbf{X}}_{d}$ the desired MP twist, $\mathbf{q}_{d}$ the 8dimensional vector of desired angular positions of the motors, $\tau_{m}$ the 8-dimensional vector of desired motor torques. $K_{p}$ and $K_{d}$ are the proportional and derivative gains of the controller, respectively, and have been tuned identically for all motors such that the robot achieves acceptable accuracy and stability with the MP alone.

While a PD controller is the basis of robot control, a feedforward term is commonly included in CDPR control strategies [17], [18] to predict the dynamics of the platform 
and improve the robot accuracy. Based on the dynamic model, it is possible to add a term to the controller that will anticipate the MP dynamics and compensate for the payload mass along the trajectory. The final controller is denoted as (PDFF), as is includes the feedforward term calculated in real-time.

From (12), this feedforward term is defined as

$$
\tau_{f f}=\frac{m r \mathbf{W}^{+}\left[\ddot{\mathbf{X}}_{d}+\mathbf{g}\right]}{R}
$$

with $\tau_{f f}$ the feedforward torque, $m$ the total mass of the MP and its payload, $R$ the gearbox ratio, $r$ the drum radius. $\mathbf{W}^{+}$ denotes the Moore-Penrose pseudo-inverse of $\mathbf{W}$. The MP Cartesian and angular acceleration $\ddot{\mathbf{X}}_{d}$ has been considered to anticipate the robot dynamics.

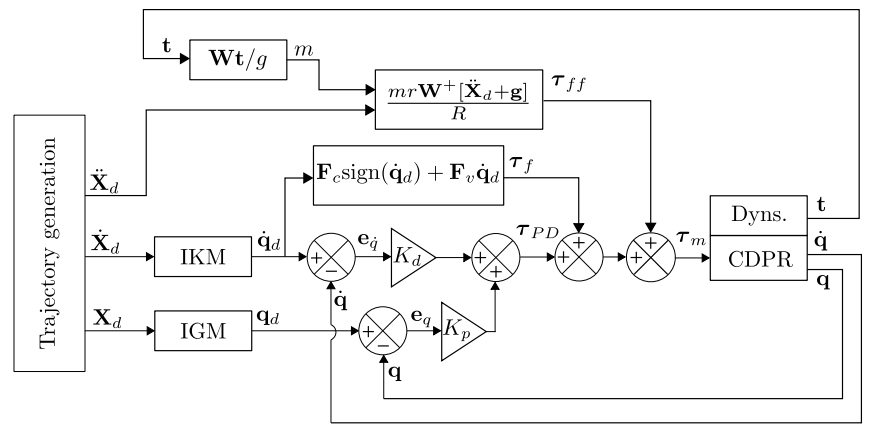

Fig. 6: Control scheme with feedforward for real time mass estimation and compensation (PDFF).

The inverse geometric model (IGM) and inverse kinematic model (IKM) in the experiments used Model I. Model II will be used in the future experimental tests and should lead to better accuracy. A friction compensation was implemented in each actuation chain to compensate the losses in the transmission systems:

$$
\tau_{f}=\mathbf{F}_{c} \operatorname{sign}\left(\dot{\mathbf{q}}_{d}\right)+\mathbf{F}_{v} \dot{\mathbf{q}}_{d},
$$

with $\tau_{f}$ the generated friction compensation torque and $\dot{\mathbf{q}}_{d}$ the desired motor rate vector. $\mathbf{F}_{c}$ and $\mathbf{F}_{v}$ are respectively the vectors containing the static and viscous friction coefficients separately identified for each motor.

\section{SENSITIVITy ANALYSis BASED ON EXPERIMENTAL RESULTS}

This section analyzes the effect of the pulleys geometric parameters onto the payload estimation as well as the comparison between force sensors and motor currents for cable tensions and payload estimation. Moreover, the performance of the controller described in Sec. IV are presented thereafter.

\section{A. Pulley effect onto payload estimation}

The study of the effect of the pulleys geometry on the payload estimation is realized along the test trajectory described in Sec. II.C using the cable tensions measured by the dynamometers. Since we want to establish a method that is compatible with real-time computation, the wrench matrix of the platform is computed using the desired poses of the platform. If the processor can solve the direct geometric model fast enough, the mass estimation from the estimated position will be further improved.

Figure 7 presents the estimated mass of the payload along the trajectory for the three different payloads with both the basic model, Model I, and the model including the geometry of the pulleys, Model II. It is noteworthy that the payload mass estimation is better with the model including the geometry of the pulleys than with the model which does not consider the pulleys. The changes in the estimation can be explained by the new wrench matrix and computed cable angles from the MP to the exit points on the pulleys. This leads to higher and more stable values for the mass estimations along the trajectory. As shown in Tab. I and Figs. 8 to 9, the average mass estimation error drops from $4.5 \%$ to around $1 \%$ when considering the pulleys and the standard deviation is reduced by more than $20 \%$. The variations in the estimation from $t=0 \mathrm{~s}$ to $t=5 \mathrm{~s}, t=25 \mathrm{~s}$ to $t=35 \mathrm{~s}$ and $t=55 \mathrm{~s}$ to $t=60 \mathrm{~s}$ are due to the acceleration of the MP in the upward and downward phases and to vibrations which caused cable tensions fluctuations.

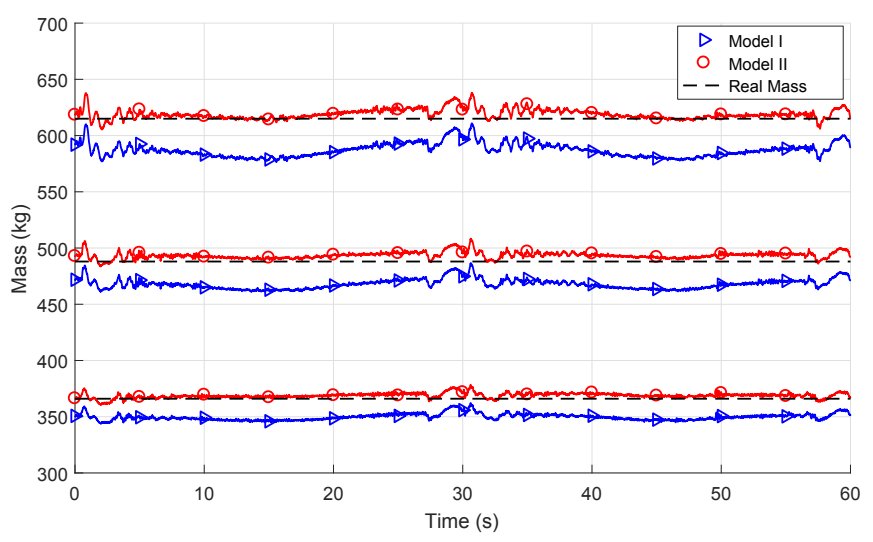

Fig. 7: Estimated mass (kg) with the two models defined in Sec. III.A and III.B and for the three payloads along the test trajectory; the cable tensions are measured with dynamometers.

TABLE I: Estimated mass along the test trajectory with Models I and II.

\begin{tabular}{r|ccc}
\hline \hline Model & MP & MP+M & MP+M \\
\hline Mean mass (kg) & & & \\
Real mass & 366 & 488 & 615 \\
Model I & 349.8 & 468.0 & 587.1 \\
Model II & 368.9 & 493.5 & 619.1 \\
\hline Error (\%) & & & \\
Model I & 4.4 & 4.1 & 4.5 \\
Model II & 0.8 & 1.1 & 0.7 \\
\hline Standard deviation (kg) & & & \\
Model I & 2.8 & 4.3 & 6.2 \\
Model II & 2.2 & 3.0 & 4.3 \\
\hline \hline
\end{tabular}

The position of the center of mass along the x-axis and the $\mathrm{y}$-axis of the MP frame $\mathscr{F}_{p}$ have been calculated with 


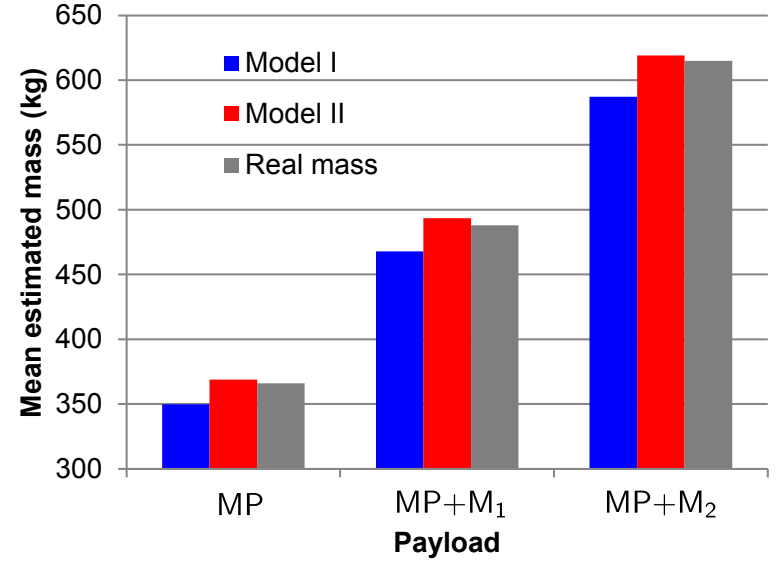

Fig. 8: Mean of the estimated mass along the trajectory with the two models defined in Sec. III.A and III.B and the three payloads.

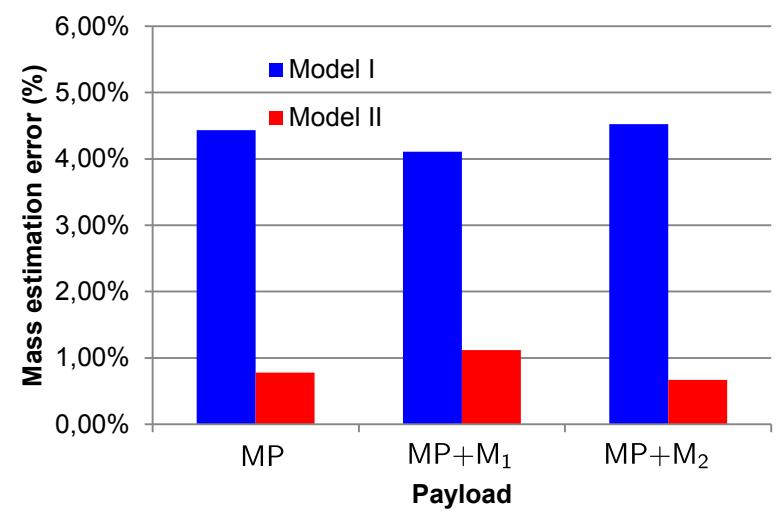

Fig. 9: Mean of mass estimation error along the test trajectory with Models I and II and three payloads.

Models I and II and are illustrated in Fig. 10 for the heaviest payload, i.e., with mass $\mathrm{M}_{2}$. From Fig. 10, we can notice that there is an offset of the center of mass of the set composed of the moving-platform MP and the metal plate $\mathrm{M}_{2}$, which is consistent with the real position of payload $\mathrm{M}_{2}$ under the moving-platform as shown in Fig. 11.

According to the complexity of the pulleys model, its use in the mass estimation is therefore of interest and Model II is used in the remainder of the paper.

\section{B. Tension estimations versus dynamometer measurements}

Parallel robots are not all equipped with dynamometers, since the integration of additional sensors might be expensive. However, it may be essential to implement a payload compensation method by estimating the cable tensions. In this section, the influence of the cable tensions measurements on the payload estimation is studied. The experiments were made with the heaviest payload, i.e. $\mathrm{MP}+\mathrm{M}_{2}$ of $615 \mathrm{~kg}$, as illustrated in Fig. 11.

Motor torques, obtained from the motors currents, can be used to estimate the cable tensions, knowing the gearbox ratio and the drum radius. Figure 12 shows $(i)$ the cable 1 tension measured by the dynamometer located between the

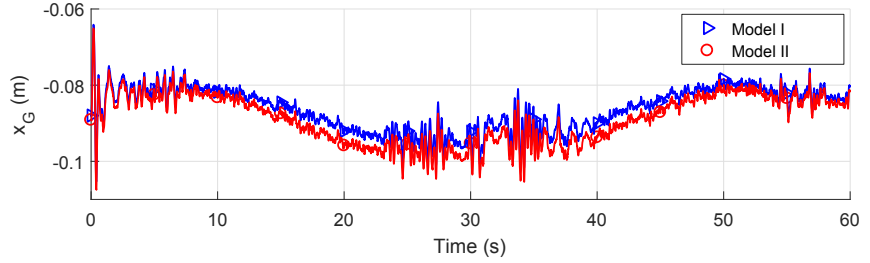

(a) $x_{G}$ Cartesian coordinate

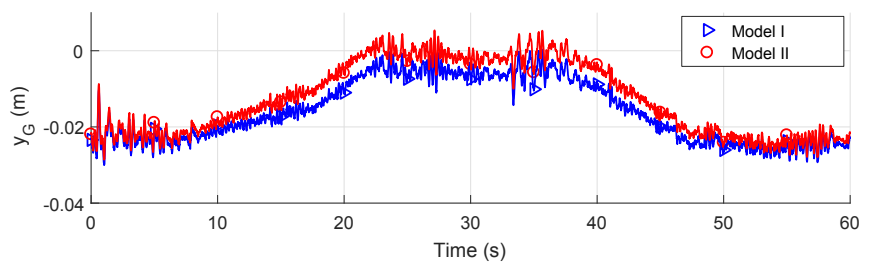

(b) $y_{G}$ Cartesian coordinate

Fig. 10: Center of mass position estimation in frame $\mathscr{F}_{p}$ for the heaviest payload $\mathrm{MP}+\mathrm{M}_{2}(615 \mathrm{~kg})$ along the test trajectory with Models I and II (Top). along X-axis (Bottom). along y-axis.

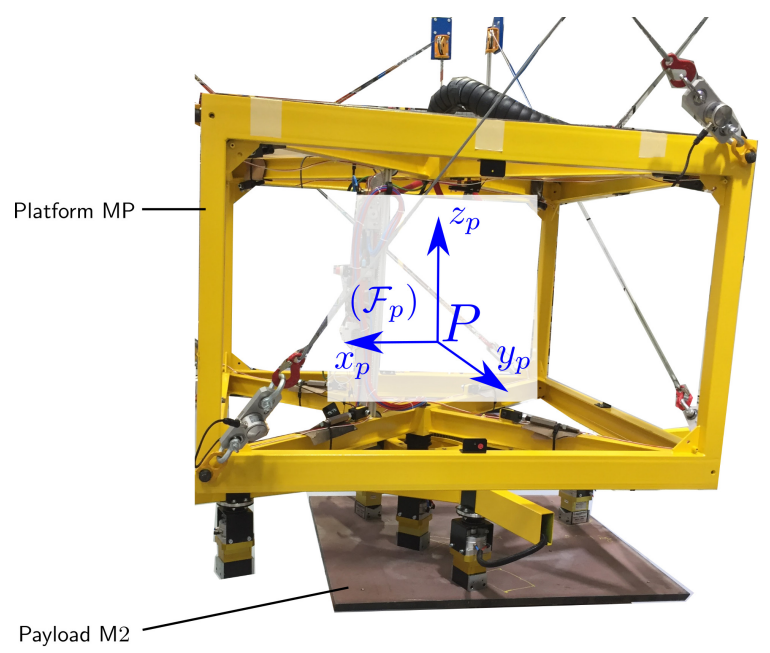

Fig. 11: Moving platform (MP) displacing payload $\mathrm{M}_{2}$. There is an offset between the center of mass of $\mathrm{M}_{2}$ and axis $z_{p}$ along axis $x_{p}$

cable 1 exit point and the MP; (ii) the cable 1 tension assessed from the raw motor 1 torque; (iii) the cable 1 tension estimated from the raw motor 1 torque and the friction model expressed in (15), to reduce the perturbation caused by friction on the measure.

It first appears that the dynamometer offers a measure with much less noise that the estimations from the raw motor torque. However, we can observe that the estimation from the motor torque and the friction model is very close to the dynamometer measurement, especially from $t=5 \mathrm{~s}$ to $t=$ $25 \mathrm{~s}$ and $t=35 \mathrm{~s}$ to $t=55 \mathrm{~s}$ when the velocity of the platform is almost constant. The discontinuities in cable 1 tension profile obtained from the motor 1 torque are due to changes of motor 1 rotation direction. These results confirm that the dynamometers offer a cleaner measure of tension, but also 


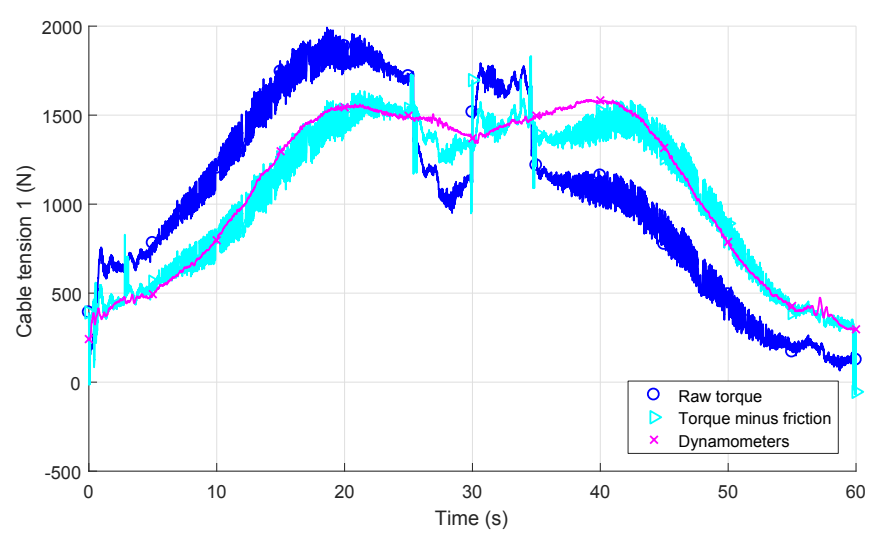

Fig. 12: Cable tension $1(\mathrm{~N})$ with dynamometers measurements, raw motor torque and torque minus frictions, for the heaviest payload $\mathrm{MP}+\mathrm{M}_{2}(615 \mathrm{~kg})$ along the test trajectory.

highlight the possibility that with the proper filter and a better identification of the friction in the motors, motor torques could give a usable reading of the cable tension.

The mass estimation along the trajectory shown in Fig. 13 shows again that the estimation is the most accurate with the dynamometer measurements. The impact of the noise in the cable tension estimation from motor torques is amplified in the mass estimation, as seen in Table II. While the mean of the estimated mass error is smaller than $6 \%$ with both the raw torques and the torques minus the friction, the maximum error reaches respectively $57 \%$ and $30 \%$ of the payload mass with those measures. On the other hand the dynamometers lead to a maximum error slightly above $4 \%$. The largest errors occur along the vertical motion of the platform, in the acceleration phases and especially when the motors direction changes, for which the mass estimation error is suddenly inverted. In the central sections of the paths the mass estimation from the torques is closer to the dynamometers results.

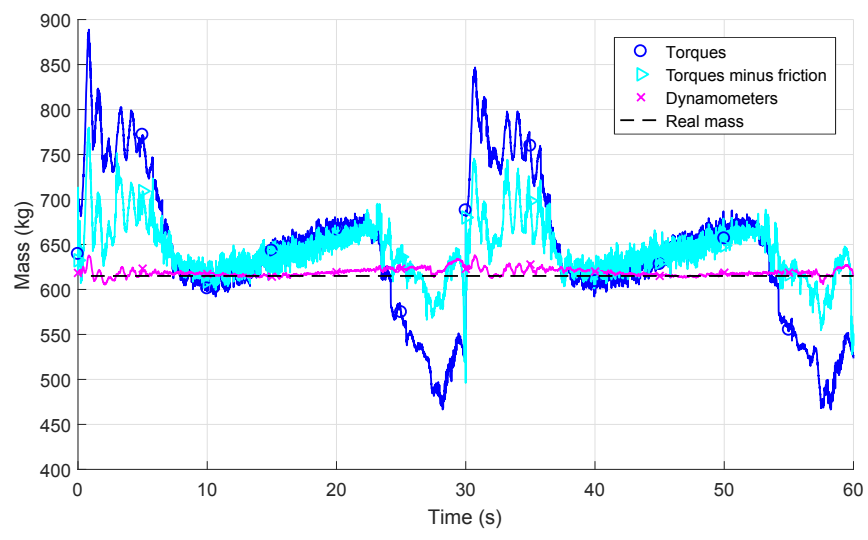

Fig. 13: Estimated mass $(\mathrm{kg})$ along the trajectory from dynamometers measures, raw motor torques and motors torques minus friction model, for the heaviest payload $\mathrm{MP}+\mathrm{M}_{2}(615$ $\mathrm{kg})$

Finally, the center of mass is estimated for payload
TABLE II: Estimated mass along the test trajectory with dynamometer measurements and motor torques.

\begin{tabular}{c|ccc}
\hline \hline Measurements & MP & MP+M & MP+M \\
\hline Real mass (kg) & 366 & 488 & 615 \\
\hline Mean mass (kg) & & & \\
Raw torques & 387.8 & 515.0 & 643.8 \\
Torques minus friction & 387.7 & 466.3 & 643.7 \\
Dynamometers & 368.8 & 493.4 & 619.1 \\
\hline Mean error (\%) & & & \\
Raw torques & 5.97 & 5.54 & 4.7 \\
Torques minus friction & 5.93 & 5.51 & 4.7 \\
Dynamometers & 0.8 & 1.1 & 0.7 \\
\hline Maximum error (\%) & & & \\
Raw torques & 56.58 & 48.1 & 44.5 \\
Torques minus friction & 30.1 & 28.1 & 26.8 \\
Dynamometers & 3.4 & 4.2 & 3.7 \\
\hline Standard deviation (kg) & & & \\
Raw torques & 66.5 & 71.9 & 78.5 \\
Torques minus friction & 20.2 & 25.1 & 31.3 \\
Dynamometers & 2.2 & 3.0 & 4.3 \\
\hline \hline
\end{tabular}

$\mathrm{MP}+\mathrm{M}_{2}$ and shown in Fig. 14. Here only the estimations from the dynamometers to the motors torques minus friction are compared since it consistently offered better results than the raw torques. The same conclusions can be made than with the mass estimation as a more stable estimation was obtained from the dynamometer measurements than with the torque, but with a fairly similar shape. A filter might lead to usable readings in the absence of force sensors.

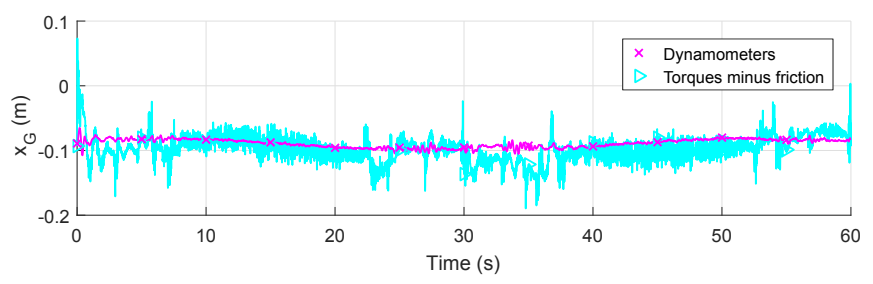

(a) $x_{G}$ Cartesian coordinate

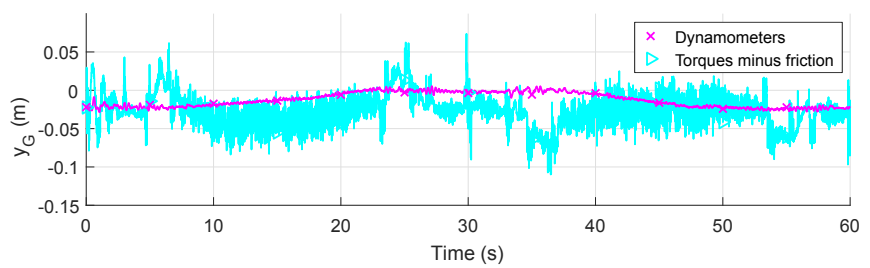

(b) $y_{G}$ Cartesian coordinate

Fig. 14: Center of mass position estimation in frame $\mathscr{F}_{p}$ for the heaviest payload $\mathrm{MP}+\mathrm{M}_{2}(615 \mathrm{~kg})$ along the test trajectory from dynamometer measurements and motor torques minus frictions (Top). along $\mathrm{x}$-axis (Bottom). along $\mathrm{y}$-axis.

\section{Control method performance}

Experiments have been realized on the CAROCA prototype along the test trajectory described in Sec. II-C with two controllers:

1) the PD controller presented in Fig. 6, with real-time update of the mass in the feedforward term (PDFF)

2) a PD controller without mass compensation (PD) 
For this comparison both controllers used the same gains $K_{p}$ and $K_{d}$. Position errors were computed by solving the direct geometric model including the pulleys with the Least Square Method. The MP position errors along the test trajectory with the two controllers and the three payloads are shown in Fig. 15. As the impact of the payload mass is directed along the gravity, we focus on the platform position accuracy along the z-axis. The PD controller suffers from an immediate error at the beginning of the trajectory, as the initial output torque of the PD is null and the platform position drops under the load. Between the lightest and the heaviest payloads, the errors of the PD controller increased up to $20 \mathrm{~mm}$. On the other hand, the PDFF controller has an absolute position error smaller than $5 \mathrm{~mm}$, for every payload. Although there are still some variations in the position accuracy, this behavior is stable enough to pick and place the heaviest metal plate, shown in Fig. 11, with a good accuracy. As a conclusion, while the performance of the PD controller are affected by the mass increment, the real time mass compensation allows for a more precise control of the platform position.
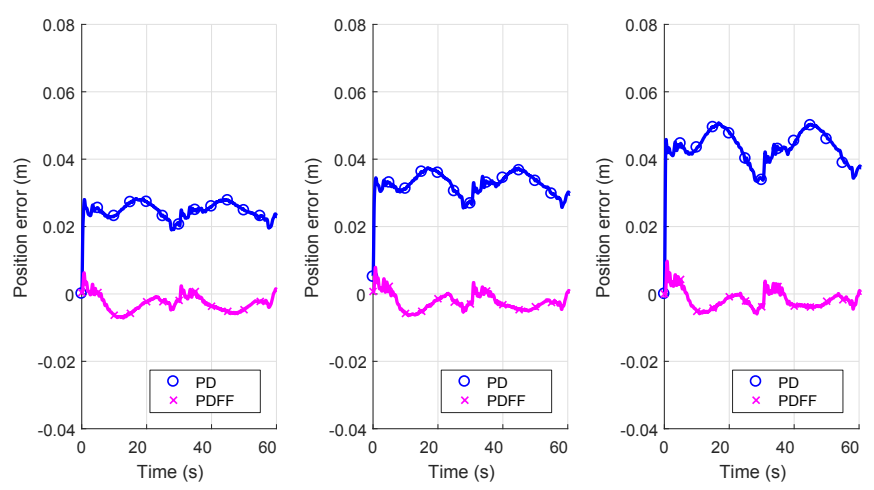

Fig. 15: Platform position error along z-axis versus time obtained with the two controllers, for three masses. Left. MP (366 kg). Center. $\mathrm{MP}+\mathrm{M}_{1}(488 \mathrm{~kg})$. Right. $\mathrm{MP}+\mathrm{M}_{2}$ (615 kg).

\section{Conclusion}

This paper dealt with the pulleys and force sensors influence on payload estimation of cable-driven parallel robots. First, the effect of the pulleys to the payload estimation was experimentally evaluated. Then, a comparison between the force sensors and the information coming from the motor currents to assess the cable tensions was made. It should be noted that the consideration of the pulleys into the geometric model of the robot substantially improves the mass and center of mass estimations of the payload at a low computational cost. Moreover, the cable tension values are much more accurate when they are measured with dynamometers instead of being assessed from the motor torques. A better friction model of the transmission systems and a filter are required to get a better estimation of the cable tensions from the motor currents. Besides, a torque controller with a feedforward term for real-time mass compensation was proposed and implemented on a CDPR prototype located at IRT Jules Verne. The experimental results confirm the interest in payload estimation for CDPR robust control. The consideration of cable elasticity and the determination of an improved friction model for the CDPR transmission systems are part of our future work.

\section{ACKNOWLEDGMENTS}

The authors wish to associate the industrial and academic partners of this project, namely, STX France, Clemessy, B\&R Automation, Clarté, IRT Jules Verne and CNRS.

\section{REFERENCES}

[1] L. Gagliardini, S. Caro, M. Gouttefarde, and A. Girin, "Discrete reconfiguration planning for Cable-Driven Parallel Robots," Mechanism and Machine Theory, vol. 100, pp. 313-337, 2016.

[2] C. Holland and D. Cannon, "Cable array robot for material handling," US Patent 6,826,452, vol. 1, no. 12, 2004.

[3] M. Hassan and A. Khajepour, "Analysis of a Large-Workspace CableActuated Manipulator for Warehousing Applications," in Volume 7: 33rd Mechanisms and Robotics Conference, Parts A and B, 2009, pp. $45-53$.

[4] A. Pott, C. Meyer, and A. Verl, "Large-scale assembly of solar power plants with parallel cable robots," Isr/Robotik 2010, pp. 999-1004, 2010.

[5] S. Kawamura, H. Kino, and C. Won, "High-speed manipulation by using parallel wire-driven robots," Robotica, vol. 18, no. 1, p. S0263574799002477, 2000.

[6] A. Fortin-Coté, P. Cardou, and C. Gosselin, "An admittance control scheme for haptic interfaces based on cable-driven parallel mechanisms," in Proceedings - IEEE International Conference on Robotics and Automation, 2014, pp. 819-825.

[7] R. Yao, X. Tang, J. Wang, and P. Huang, "Dimensional optimization design of the four-cable-driven parallel manipulator in fast," IEEE/ASME Transactions on Mechatronics, vol. 15, no. 6, pp. 932941, 2010.

[8] J. P. Merlet and D. Daney, "A portable, modular parallel wire crane for rescue operations," in Proceedings - IEEE International Conference on Robotics and Automation, 2010, pp. 2834-2839.

[9] W. Kraus, V. Schmidt, P. Rajendra, and A. Pott, "Load identification and compensation for a Cable-Driven parallel robot," in Proceedings - IEEE International Conference on Robotics and Automation, 2013, pp. 2485-2490.

[10] S. Baklouti, E. Courteille, S. Caro, and M. Dkhil, "Dynamic and Oscillatory Motions of Cable-Driven Parallel Robots Based on a Nonlinear Cable Tension Model," Journal of Mechanisms and Robotics, vol. 9, no. 6, p. 061014, 2017.

[11] L. Gagliardini, "Discrete reconfigurations of cable-driven parallel robots," Ph.D. dissertation, 2016.

[12] A. Pott, "Influence of Pulley Kinematics on Cable-Driven Parallel Robots," Latest Advances in Robot Kinematics, pp. 197 -204, 2012.

[13] C. Schenk, C. Masone, A. Pott, and H. H. Bülthoff, "Application of a differentiator-based adaptive super-twisting controller for a redundant cable-driven parallel robot," in Mechanisms and Machine Science, vol. 53, 2018, pp. 254-267.

[14] C. Germain, S. Caro, S. Briot, and P. Wenger, "Optimal design of the irsbot-2 based on an optimized test trajectory," in ASME 2013 International Design Engineering Technical Conferences and Computers and Information in Engineering Conference. American Society of Mechanical Engineers, 2013, pp. V06AT07A056-V06AT07A056.

[15] L. Gagliardini, M. Gouttefarde, and S. Caro, "Determination of a dynamic feasible workspace for cable-driven parallel robots," in Advances in Robot Kinematics 2016. Springer, 2018, pp. 361-370.

[16] S. Kawamura, H. Kino, and C. Won, "High-speed manipulation by using parallel wire-driven robots," Robotica, vol. 18, no. 1, p. S0263574799002477, 2000.

[17] J. Lamaury, M. Gouttefarde, A. Chemori, and P. E. Herve, "Dual-space adaptive control of redundantly actuated cable-driven parallel robots," IEEE International Conference on Intelligent Robots and Systems, pp. 4879-4886, 2013.

[18] A. Vafaei, M. M. Aref, and H. D. Taghirad, "Integrated controller for an over-constrained cable driven parallel manipulator: KNTU CDRPM," in Proceedings - IEEE International Conference on Robotics and Automation, 2010, pp. 650-655. 